\title{
Shedding of Marburg Virus in Naturally Infected Egyptian Rousette Bats, South Africa, 2017
}

Janusz T. Pawęska, Nadia Storm, Wanda Markotter, Nicholas Di Paola, Michael R. Wiley, Gustavo Palacios, Petrus Jansen van Vuren

We detected Marburg virus RNA in rectal swab samples from Egyptian rousette bats in South Africa in 2017. This finding signifies that fecal contamination of natural bat habitats is a potential source of infection for humans. Identified genetic sequences are closely related to Ravn virus, implying wider distribution of Marburg virus in Africa.

The genus Marburgvirus, family Filoviridae, com1 prises 1 species, Marburg marburgvirus, which comprises 2 marburgviruses, Marburg virus (MARV) and Ravn virus (RAVV) (1). Marburgviruses cause sporadic but often fatal MARV disease in humans and nonhuman primates (2). The Egyptian rousette bat (Rousettus aegyptiacus) has been implicated as the primary reservoir for marburgviruses (3-9), but the mechanisms by which they are maintained in these bats remain elusive. Evidence of marburgvirus circulation was reported from countries where MARV disease outbreaks have not been recorded (10-12). Determining the risks for spread and developing evidence-based public health strategies to prevent zoonotic transmission requires up-to-date knowledge about marburgvirus geographic range; genetic diversity; and transmission mechanisms, including natural ports of entry and shedding patterns. To clarify which marburgviruses are circulating and how they

Author affiliations: National Institute for Communicable Diseases of the National Health Laboratory Service, Johannesburg, South Africa (J.T. Pawęska, N. Storm, P. Jansen van Vuren); Boston University, Boston, Massachusetts, USA (N. Storm); University of Pretoria, Pretoria, South Africa (J.T. Pawęska, W. Markotter); University of Nebraska Medical Center, Omaha, Nebraska, USA (N. Di Paola, M.R. Wiley, G. Palacios); US Army Medical Research Institute of Infectious Diseases, Frederick, Maryland, USA (N. Di Paola, M.R. Wiley, G. Palacios); Australian Centre for Disease Preparedness, Commonwealth Scientific and Industrial Research Organisation-Health and Biosecurity, Geelong, Victoria, Australia (P. Jansen van Vuren)

DOI: https://doi.org/10.3201/eid2612.202108 are maintained in Egyptian rousette bat populations in South Africa, we tested oral and rectal swab samples and blood samples collected during a previously identified peak season of marburgvirus transmission in a local Egyptian rousette bat population (13).

\section{The Study}

We conducted this work in accordance with approved protocols by animal ethics committees of the National Health Laboratory Service (Johannesburg, South Africa; AEC 137/12) and the University of Pretoria (Pretoria, South Africa; EC054-14). During February-November 2017, a total of 1,674 Egyptian rousette bats (February, 107 bats; April, 600; May, 563; September, 214; November, 190) were captured, aged, and sampled at Matlapitsi Cave, Limpopo Province, South Africa, as described previously (13). We collected and processed oral and rectal swab samples from each bat and blood from a subset of 423 bats, as described previously (7). Swab samples were collected and pooled by aliquoting $4 \times 25 \mu \mathrm{L}$ of the media of each sample into a microcentrifuge tube, yielding a total of 416 pools containing $100 \mu \mathrm{L}$ of pooled rectal swab samples and 4 pools containing 50 or $75 \mu \mathrm{L}$ of pooled oral swab samples. We conducted serologic, virologic, and molecular tests as described previously (7). In addition, we conducted real-time quantitative reverse transcription PCR (qRT-PCR) for individual swab samples when the pool tested positive. We prepared sequencing libraries using the TruSeq RNA Access Kit (Illumina, https:/ / www.illumina.com) with MARV-specific bait enrichment, followed by sequencing on an Illumina NextSeq, genomic alignment and phylogenetic analysis (13). We calculated the significance of differences in several positive swab samples and seropositivity using the Fisher exact test in Stata version 13 (StataCorp, https:/ / www.stata.com).

Seven rectal swab pools (5 from April 2017 and 2 from September 2017) were qRT-PCR positive; the remaining rectal swab samples collected during 
February-November 2017 were all negative. The number of individual positive rectal swab samples ranged from 1 to 3 per positive pool, totaling 11 positive samples. Only 1 oral swab sample pool, from April 2017, yielded a positive qRT-PCR result, containing a single positive oral swab sample (Table 1). Of 600 rectal swab samples collected during 3 nights in April, 9 (1.5\%) were positive; of 215 rectal swab samples collected during 2 nights in September, 2 (0.9\%) were positive. We found no significant difference between the number of positive rectal swab samples collected in April and the number collected September. The number of positive rectal swab samples differed significantly from the number of positive oral swab samples collected in April $(p=0.02)$. Attempts to culture marburgvirus from qRT-PCR-positive swab samples were unsuccessful. Identical results from specimens with cycle threshold $\left(C_{t}\right)>30$ were obtained in other studies $(6,13)$.

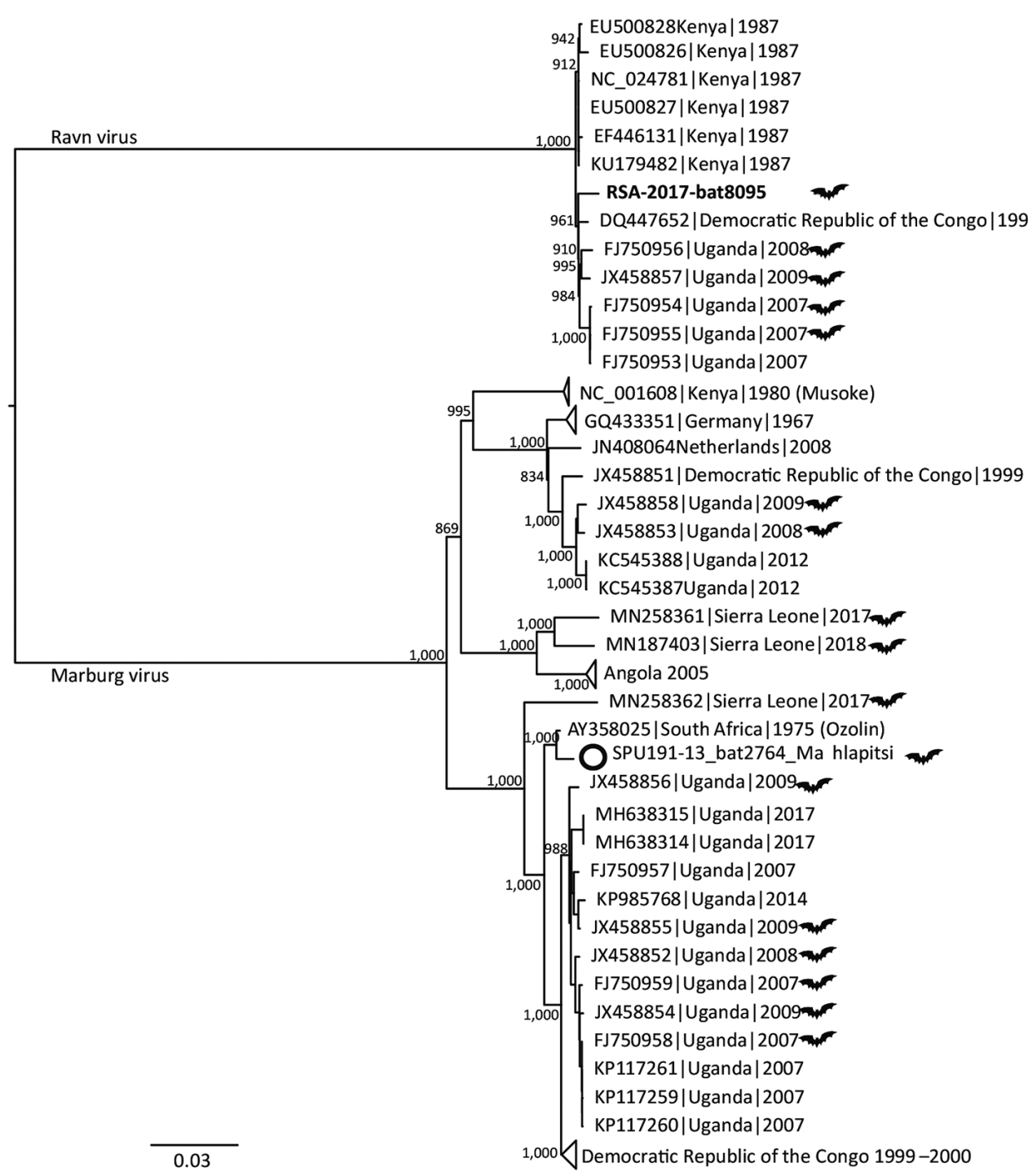
November $2017^{*}$
Table 1. qRT-PCR results of oral and rectal swab samples from juvenile Egyptian rousette bats (Rousettus aegyptiacus) at Matlapitsi Cave, Limpopo Province, South Africa, February-

\begin{tabular}{|c|c|c|c|c|}
\hline \multirow[b]{2}{*}{ Bat ID } & \multirow[b]{2}{*}{ Capture date } & \multirow[b]{2}{*}{ Sex } & \multicolumn{2}{|c|}{$\begin{array}{l}\text { Swab sample type and } \\
\text { qRT-PCR result, } C_{t} \dagger\end{array}$} \\
\hline & & & Oral & Rectal \\
\hline Apr-77 & Apr 4 & $\mathrm{~F}$ & Negative & 30.61 \\
\hline Apr-117 & Apr 4 & $\mathrm{~F}$ & Negative & 30.57 \\
\hline Apr-118 & Apr 4 & $F$ & Negative & 30.95 \\
\hline Apr-124 & Apr 4 & $F$ & Negative & 30.66 \\
\hline Apr-133 & Apr 4 & $\mathrm{~F}$ & Negative & 30.46 \\
\hline Apr-232 & Apr 5 & $\mathrm{~F}$ & 33.01 & Negative \\
\hline Apr-369 & Apr 6 & $\mathrm{~F}$ & Negative & 31.9 \\
\hline Apr-380 & Apr 6 & M & Negative & 32.67 \\
\hline Apr-399 & Apr 6 & $\mathrm{~F}$ & Negative & 31.7 \\
\hline Apr-470 & Apr 6 & M & Negative & 31.8 \\
\hline 8025 & Sep 26 & $\mathrm{~F}$ & Negative & 31.7 \\
\hline 0895 & Sep 20 & $\mathrm{~F}$ & Negative & 33.05 \\
\hline
\end{tabular}

${ }^{*} \mathrm{C}_{\mathrm{t}}$, cycle threshold; ID, identification; qRT-PCR, quantitative real-time reverse transcription PCR.

†Negative, $C_{t}>40$.
Figure 1. Midpoint-rooted, maximum-likelihood phylogeny of complete and near-complete MARV and RAVV genomes.

Phylogenetic tree shows evolutionary relationships of marburgvirus detected in a rectal swab sample from a subadult Egyptian rousette female bat (Rousettus aegyptiacus) in Matlapisi Cave, Limpopo Province, South Africa, 2017 (black filled circle; GenBank accession no. MT321489), and reference viruses, including the SPU191-13 bat 2764 Mahlapitsi strain (white circle; GenBank accession no. MG725616), detected in the same cave in July 2013. Complete and nearcomplete genome sequences from GenBank (accession numbers indicated) were aligned with the partial MARV sequence obtained from RSA8095bat using MUSCLE version 3.8.31 (https://www.drive5.com/ muscle), and RAxML version 8.2.10 (https://cme.h-its.org/ exelixis/web/software/raxml/ index.html) was used to infer the best-scoring maximum-likelihood tree after 1,000 bootstrap replicates. Node values indicate the bootstrap support values. Genomes isolated from bats are shown using a bat symbol. Scale bar indicates nucleotide substitutions per site. MARV, Marburg virus; RAVV, Ravn virus. 


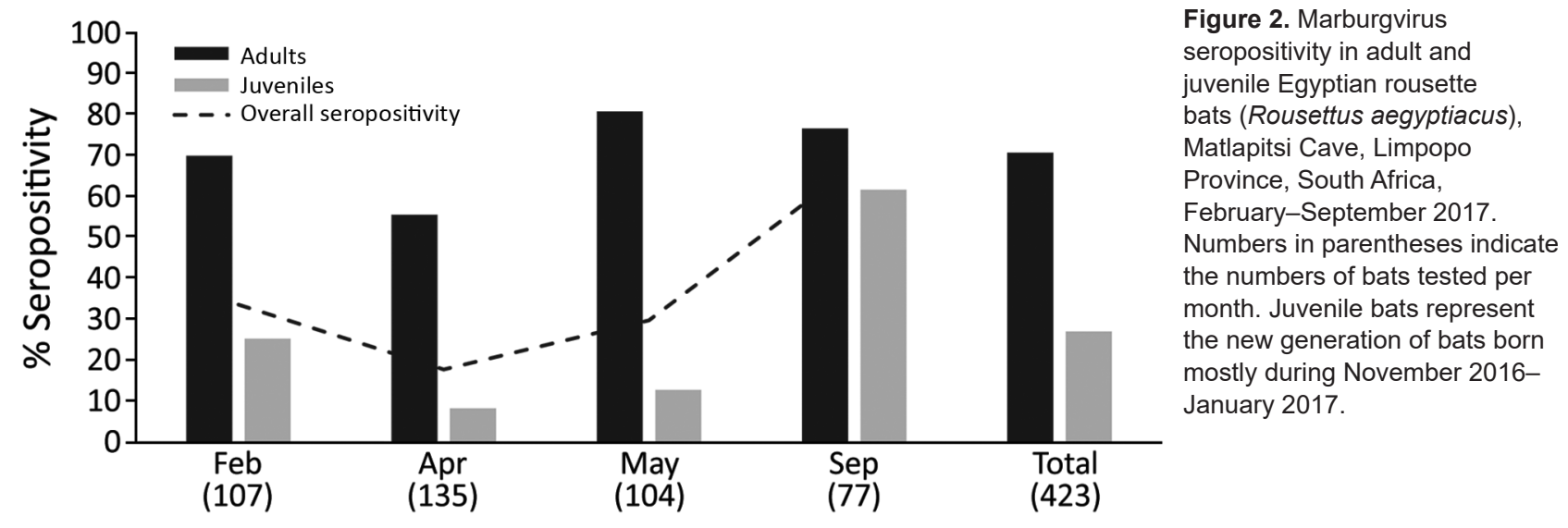

We obtained sufficient marburgvirus-specific sequence data only from 1 of the 12 individual positive swab samples for phylogenetic analysis: a rectal swab sample, collected from a juvenile female (bat 8095 ) in September 2017, from which we recovered $79.2 \%(15.1 / 19.1 \mathrm{~kb})$ of the genome. We merged sequencing reads from replicate sequencing runs and mapped 2,472 reads to the MARV reference genome. Maximum coverage per base obtained was 291 reads; some regions had no coverage. The average coverage per base across the genome was 18.5 reads (when we included 0 coverage regions), and the average coverage when we excluded 0 coverage regions was 40 reads. We obtained near-complete coding sequences for the viral protein (VP) 35 (972/990 nt; 98.2\%) and VP40 (898/912 nt; 98.5\%) genes; coverage ranged from $49.7 \%$ (VP24) to $89.3 \%$ (glycoprotein) in other open reading frames of the genome.

The marburgviruses sequence (strain RSA-2017bat8095) detected from the rectal swab sample of bat 8095 shared a common ancestor with all other RAVV complete or near-complete genome sequences, including 3 human isolates from Kenya (14), Uganda (15), and the Democratic Republic of Congo (3) and several bat isolates from Uganda (4) (Figure 1). The RSA-2017-bat8095 nt sequence shared $\approx 77 \%$ identity with the MARV SPU191-13bat2764 Mahlapitsi strain (GenBank accession no. MG725616) that was collected and characterized from Matlapitsi Cave 4 years earlier.

Of 423 bats tested, 143 (33.8\%) were positive for antibodies against marburgviruses (73 adults and 70 juveniles). Lowest overall seroprevalence occurred in April 2017 (17.78\%) and ranged from 8.3\% in juveniles $(9 / 108)$ to $55.6 \%$ in adults $(15 / 27)$ (Figure 2 ). Overall seropositivity did not differ significantly between male and female bats, but the overall seropositivity differed significantly between juvenile (forearm
$<89 \mathrm{~mm}$; <1-year-old) and adult bats $(\mathrm{p}=0.03)$. We detected seroconversion in $6(33.3 \%)$ of 18 recaptured bats (Table 2).

\section{Conclusions}

The period of the lowest seropositivity in juveniles (April-May) resulting in the highest number of potentially susceptible bats at Matlapitsi Cave was the same as previously identified (13). This finding coincides with demonstrable seroconversions and virus shedding and represents a period of increased exposure. The significantly higher number of marburgvirus-positive rectal than oral swab samples we detected contrasts with results from experimentally infected Egyptian rousette bats and field studies in Uganda $(4,8,9)$. Experimental data on marburgvirus shedding were obtained from subcutaneously inocWhether this mode of infection represents a natural portal of entry for marburgviruses in Egyptian rousette bats and to what extent viral shedding patterns in colonized bats can be extrapolated to natural settings are unknown.

Table 2. Marburgvirus seroconversion in 6 of 18 Egyptian rousette bats (Rousettus aegyptiacus) recaptured at Matlapitsi Cave, Limpopo Province, South Africa, February 2017September $2017^{*}$

\begin{tabular}{lccccc}
\hline & \multicolumn{2}{c}{ First capture } & & \multicolumn{2}{c}{ Recapture } \\
\cline { 2 - 3 } \cline { 5 - 6 } Bat ID & $\begin{array}{c}\text { iELISA, } \\
\% \dagger\end{array}$ & $\begin{array}{c}\text { Capture } \\
\text { date }\end{array}$ & & $\begin{array}{c}\text { iELISA, } \\
\% \dagger\end{array}$ & $\begin{array}{c}\text { Capture } \\
\text { date }\end{array}$ \\
\hline SMB676 & 2.2 & $2016 \mathrm{Apr}$ & & 23.3 & $2017 \mathrm{Feb}$ \\
SMB797 & 12.4 & $2016 \mathrm{Jun}$ & & 23.7 & $2017 \mathrm{Apr}$ \\
SMA780 & 0.7 & $2014 \mathrm{Jul}$ & & 61.7 & $2017 \mathrm{Sep}$ \\
SM906 & 15.2 & $2013 \mathrm{Sep}$ & & 202.4 & $2017 \mathrm{Sep}$ \\
SMB160 & 2.5 & $2015 \mathrm{Mar}$ & & 51.3 & $2017 \mathrm{Sep}$ \\
SMB978 & 11.1 & 2016 Nov & & 142.2 & $2017 \mathrm{Sep}$ \\
\hline *Recaptured bats were identified by a previously applied unique tattoo \\
number. iELISA, indirect ELISA. & & & \\
†Percent positivity of the internal positive control serum in I-ELISA \\
calculated as (average optical density of the test serum replicates/average \\
optical density of the positive control serum replicates) $\times 100 ;$ cutoff \\
percent positivity of iELISA = 16.78\% (7).
\end{tabular}
ulated and colonized Egyptian rousette bats (7-9). 
Our findings highlight the risk for marburgvirus fecal environmental contamination and for Egyptian rousette bat roosting sites as a possible source of virus spillover. Roosting behavior enabling direct physical contact suggests that fecaloral transmission of marburgviruses in bats can occur. Biting among animals or biting by hematophagous ectoparasites might result in inoculation of wounds with contaminated feces or exposure to contaminated fomites.

Our findings, combined with earlier detection of an Ozolin-like MARV in Egyptian rousette bats roosting at Matlapitsi Cave (13), suggest local co-circulation of multiple marburgviruses genetic variants. Detection of RAVV in South Africa, closely related to East African isolates, indicates that long-distance movement of Egyptian rousette bats contributes to widespread geographic dispersion of marburgviruses. Moreover, it implies that more virulent strains, such as the MARV Angolan strain (2), might be co-circulating. Entering caves and mining have been associated with MARV spillover (3$6,14,15)$ and detection of viral RNA in rectal swab samples, highlight the potential route of transmission. Confirmation of the period for the highest virus exposure risk further highlights the value of biosurveillance and demonstrates that marburgviruses continue endemic circulation in South Africa. This circulation represents a potential threat that needs to be communicated to at-risk communities as a part of evidence-based public health education and prevention of pathogen spillover.

\section{Acknowledgments}

We thank the students of the Centre for Viral Zoonoses, University of Pretoria, and the staff of the Center for Emerging Zoonotic and Parasitic Diseases, National Institute for Communicable Diseases of the National Health Laboratory Service, for technical assistance during field work. We also thank the Ga Mafefe community in the Limpopo Province for supporting our research at Matlapitsi Cave.

This work was supported by the South African National Research Foundation (grant no. UID 98339); the Poliomyelitis Research Foundation (grant no.12/14); the Division of Global Disease Detection, Center for Global Health, US Centers for Disease Control and Prevention, under Cooperative Agreement no. 5, NU2GGH001874-02-00; the US Defense Threat Reduction Agency (CB10246); and the US Defense Biological Product Assurance Office through task order award (FA4600-12-D-9000).

\section{About the Author}

Dr. Paweska is head of the Center for Emerging Zoonotic and Parasitic Diseases at the National Institute for Communicable Diseases, Johannesburg, South Africa. His research interests include diagnostic testing, epidemiology, and ecology of Biosafety Level 4 zoonotic viral agents.

\section{References}

1. Amarasinghe GK, Aréchiga Ceballos NG, Banyard AC, Basler CF, Bavari S, Bennett AJ, et al. Taxonomy of the order Mononegavirales: update 2018. Arch Virol. 2018;163:2283-94. https://doi.org/10.1007/s00705-018-3814-x

2. Amman BR, Swanepoel R, Nichol ST, Towner JS. Ecology of filoviruses. Curr Top Microbiol Immunol. 2017;411:23-61. https://doi.org/10.1007/82_2017_10

3. Bausch DG, Nichol ST, Muyembe-Tamfum JJ, Borchert M, Rollin PE, Sleurs H, et al.; International Scientific and Technical Committee for Marburg Hemorrhagic Fever Control in the Democratic Republic of the Congo. Marburg hemorrhagic fever associated with multiple genetic lineages of virus. N Engl J Med. 2006;355:909-19. https:/ / doi.org/ 10.1056/NEJMoa051465

4. Towner JS, Amman BR, Sealy TK, Carroll SAR, Comer JA, Kemp A, et al. Isolation of genetically diverse Marburg viruses from Egyptian fruit bats. PLoS Pathog. 2009; 5:e1000536. https://doi.org/10.1371/journal.ppat.1000536

5. Towner JS, Khristova ML, Sealy TK, Vincent MJ, Erickson BR, Bawiec DA, et al. Marburgvirus genomics and association with a large hemorrhagic fever outbreak in Angola. J Virol. 2006;80:6497-516. https:/ / doi.org/10.1128/ JVI.00069-06

6. Amman BR, Carroll SA, Reed ZD, Sealy TK, Balinandi S, Swanepoel R, et al. Seasonal pulses of Marburg virus circulation in juvenile Rousettus aegyptiacus bats coincide with periods of increased risk of human infection. PLoS Pathog. 2012;8:e1002877. https://doi.org/10.1371/ journal.ppat.1002877

7. Paweska JT, Jansen van Vuren P, Fenton KA, Graves K, Grobbelaar AA, Moolla N, et al. Lack of Marburg virus transmission from experimentally infected to susceptible in-contact Egyptian fruit bats. J Infect Dis. 2015;212(Suppl 2):S109-18. https:// doi.org/10.1093/infdis/jiv132

8. Amman BR, Jones MEB, Sealy TK, Uebelhoer LS, Schuh AJ, Bird BH, et al. Oral shedding of Marburg virus in experimentally infected Egyptian fruit bats (Rousettus aegyptiacus). J Wildl Dis. 2015;51:113-24. https://doi.org/ 10.7589/2014-08-198

9. Schuh AJ, Amman BR, Jones ME, Sealy TK, Uebelhoer LS, Spengler JR, et al. Modelling filovirus maintenance in nature by experimental transmission of Marburg virus between Egyptian rousette bats. Nat Commun. 2017;8:14446. https://doi.org/10.1038/ncomms14446

10. Towner JS, Pourrut X, Albariño CG, Nkogue CN, Bird BH, Grard G, et al. Marburg virus infection detected in a common African bat. PLoS One. 2007;2:e764. https://doi.org/ 10.1371/journal.pone.0000764

11. Kajihara M, Hang' ombe BM, Changula K, Harima H, Isono M, Okuya K, et al. Marburgvirus in Egyptian fruit bats, Zambia. Emerg Infect Dis. 2019;25:1577-80. https://doi.org/10.3201/eid2508.190268

12. Amman BR, Bird BH, Bakarr IA, Bangura J, Schuh AJ, Johnny J, et al. Isolation of Angola-like Marburg virus from 
Egyptian rousette bats from West Africa. Nat Commun. 2020;11:510. https://doi.org/10.1038/s41467-020-14327-8

13. Pawęska JT, Jansen van Vuren P, Kemp A, Storm N, Grobbelaar AA, Wiley MR, et al. Marburg virus infection in Egyptian rousette bats, South Africa, 2013-2014. Emerg Infect Dis. 2018;24:1134-7. https:// doi.org/10.3201/ eid2406.172165

14. Johnson ED, Johnson BK, Silverstein D, Tukei P, Geisbert TW, Sanchez AN, et al. Characterization of a new Marburg virus isolated from a 1987 fatal case in Kenya. Arch Virol Suppl. 1996;11:101-14. https:// doi.org/10.1007/978-3-70917482-1_10
15. Adjemian J, Farnon EC, Tschioko F, Wamala JF, Byaruhanga E, Bwire GS, et al. Outbreak of Marburg hemorrhagic fever among miners in Kamwenge and Ibanda Districts, Uganda, 2007. J Infect Dis. 2011;204(Suppl 3): S796-9. https://doi.org/10.1093/infdis/jir312

Address for correspondence: Janusz T. Pawęska, Center for Emerging Zoonotic and Parasitic Diseases, National Institute for Communicable Diseases of the National Health Laboratory Service, 1 Modderfontein Rd, Sandringham 2131, Johannesburg, South Africa: email: januszp@nicd.ac.za

\section{February 2020} Coronaviruses

- Middle East Respiratory Syndrome Coronavirus Transmission

- Acute Toxoplasmosis among Canadian Deer Hunters Associated with Consumption of Undercooked Deer

- Public Health Program for Decreasing Risk for Ebola Virus Disease Resurgence from Survivors of the 2013-2016 Outbreak, Guinea

- Characteristics of Patients with Acute Flaccid Myelitis, United States, 2015-2018

- Illness Severity in Hospitalized Influenza Patients by Virus Type and Subtype, Spain, 2010-2017

- Exposure to Ebola Virus and Risk for Infection with Malaria Parasites, Rural Gabon

- Cost-effectiveness of Screening Program for Chronic Q Fever, the Netherlands

- Unique Clindamycin-Resistant Clostridioides difficile Strain Related to Fluoroquinolone-Resistant Epidemic BI/RT027 Strain

- Porcine Deltacoronavirus Infection and Transmission in Poultry, United States

- Chronic Human Pegivirus 2 without Hepatitis C Virus Co-infection

- Interspecies Transmission of Reassortant Swine Influenza A Virus Containing Genes from Swine Influenza $\mathrm{A}(\mathrm{H} 1 \mathrm{~N} 1)$ pdm09 and $A(H 1 N 2)$ Viruses

- Use of Surveillance Outbreak Response Management and Analysis System for Human Monkeypox Outbreak, Nigeria, 2017-2019

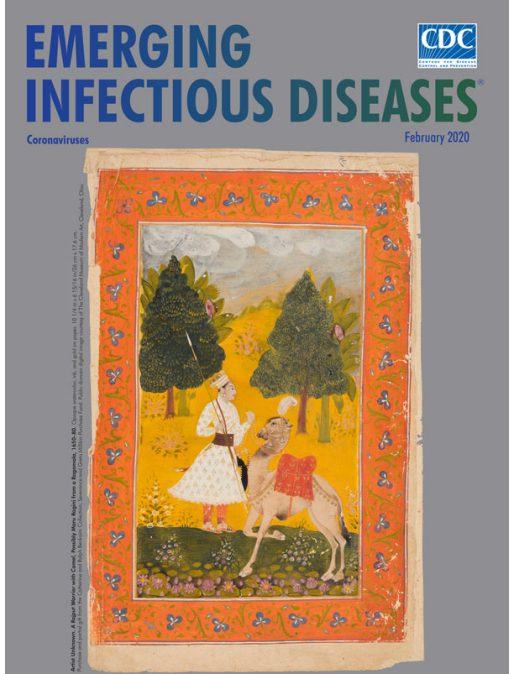

- Multiplex Mediator Displacement Loop-Mediated Isothermal Amplification for Detection of Treponema pallidum and Haemophilus ducreyi

- Novel Subclone of CarbapenemResistant Klebsiella pneumoniae Sequence Type 11 with Enhanced Virulence and Transmissibility, China

- Neutralizing Antibodies against Enteroviruses in Patients with Hand, Foot and Mouth Disease

- Influence of Rainfall on Leptospira Infection and Disease in a Tropica Urban Setting, Brazil

- Early Detection of Public Health Emergencies of International Concern through Undiagnosed Disease Reports in ProMED-Mail
- Elizabethkingia anophelis Infection in Infants, Cambodia, 2012-2018

- Global Expansion of Pacific Northwest Vibrio parahaemolyticus Sequence Type 36

- Surge in Anaplasmosis Cases in Maine, USA, 2013-2017

- Emergence of Chikungunya Virus, Pakistan, 2016-2017

- Mycoplasma genitalium Antimicrobial Resistance in Community and Sexual Health Clinic Patients, Auckland, New Zealand

- Systematic Hospital-Based Trave Screening to Assess Exposure to Zika Virus

- Ocular Spiroplasma ixodetis in Newborns, France

- Human Norovirus Infection in Dogs, Thailand

- Hepatitis E Virus in Pigs from Slaughterhouses, United States, 2017-2019

- Rapid Nanopore Whole-Genome Sequencing for Anthrax Emergency Preparedness

- Rickettsia mongolitimonae Encephalitis, Southern France, 2018

- Two Cases of Newly Characterized Neisseria Species, Brazil

- Hepatitis A Virus Genotype IB Outbreak among Internally Displaced Persons, Syria

- Rickettsia parkeri and Candidatus Rickettsia andeanae in Amblyomma maculatum Group Ticks 